\title{
Paleolithic Y-haplogroup heritage predominates in a Cretan highland plateau
}

\author{
Laisel Martinez ${ }^{1}$, Peter A Underhill ${ }^{2}$, Lev A Zhivotovsky ${ }^{3}$, Tenzin Gayden ${ }^{1}$, \\ Nicholas K Moschonas ${ }^{4}$, Cheryl-Emiliane T Chow ${ }^{2}$, Simon Conti ${ }^{2}$, Elisabetta Mamolini ${ }^{5}$, \\ L Luca Cavalli-Sforza ${ }^{2}$ and Rene J Herrera*,1
}

\begin{abstract}
${ }^{1}$ Department of Biological Sciences, Florida International University, Miami, FL, USA; ${ }^{2}$ Department of Genetics, Stanford University, Stanford, CA, USA; ${ }^{3}$ N.I. Vavilov Institute of General Genetics, Russian Academy of Sciences, Moscow, Russia; ${ }^{4}$ Department of Biology, University of Crete, Crete, Greece; ${ }^{5}$ Department of Biology, University of Ferrara, Ferrara, Italy
\end{abstract}

The island of Crete, credited by some historical scholars as a central crucible of western civilization, has been under continuous archeological investigation since the second half of the nineteenth century. In the present work, the geographic stratification of the contemporary Cretan Y-chromosome gene pool was assessed by high-resolution haplotyping to investigate the potential imprints of past colonization episodes and the population substructure. In addition to analyzing the possible geographic origins of Y-chromosome lineages in relatively accessible areas of the island, this study includes samples from the isolated interior of the Lasithi Plateau - a mountain plain located in eastern Crete. The potential significance of the results from the latter region is underscored by the possibility that this region was used as a Minoan refugium. Comparisons of Y-haplogroup frequencies among three Cretan populations as well as with published data from additional Mediterranean locations revealed significant differences in the frequency distributions of $Y$-chromosome haplogroups within the island. The most outstanding differences were observed in haplogroups $\mathrm{J} 2$ and $\mathrm{R} 1$, with the predominance of haplogroup $\mathrm{R}$ lineages in the Lasithi Plateau and of haplogroup $\mathrm{J}$ lineages in the more accessible regions of the island. Y-STR-based analyses demonstrated the close affinity that R1a1 chromosomes from the Lasithi Plateau shared with those from the Balkans, but not with those from lowland eastern Crete. In contrast, Cretan R1b microsatellite-defined haplotypes displayed more resemblance to those from Northeast Italy than to those from Turkey and the Balkans.

European Journal of Human Genetics (2007) 15, 485-493. doi:10.1038/sj.ejhg.5201769; published online 31 January 2007

Keywords: Y-chromosome; haplotype; haplogroup; Crete; Lasithi Plateau

\section{Introduction}

Referred to by some as the birthplace of western civilization, the island of Crete is one of the most extensively excavated locations in the world. Crete, which is situated

*Correspondence: Dr RJ Herrera, Department of Biological Sciences, Florida International University, University Park, Room OE304, Miami, FL 33199, USA, Tel: + 1305348 1258; Fax: + 1305348 1259; E-mail: herrerar@fiu.edu

Received 13 July 2006; revised 24 October 2006; accepted 21 November 2006; published online 31 January 2007 approximately equidistant from mainland Greece, Turkey and Libya, and linked to the first two by archipelagos of stepping-stone islands, has been influenced by periodic waves of dispersing migrants.

The first permanent inhabitants of the island are believed to have arrived around $7000 \mathrm{BC}$, possibly from Anatolia. ${ }^{1}$ This founding group was mainly composed of early Neolithic farmers who established their first settlements in the fertile lowland regions of Crete. Approximately 4000 years later, this population in part formed the basis of what 
has since been termed the Minoan culture, a pre-Hellenic Bronze-Age civilization that prospered from mercantilism and trade with other Mediterranean civilizations.

It is often argued that during the later Bronze Age, Crete received an influx of Mycenaean Greeks who controlled the island from ca. $1450 \mathrm{BC}$ until the twelveth century BC. ${ }^{2}$ The end of the Bronze Age witnessed a considerable amount of cultural disruption in Crete, for reasons that remain disputed. One result of this upheaval, whether it was caused by internal warfare or by invasion from the north, was a population movement to upland refuges. ${ }^{3}$

After the Bronze Age, Crete came under the influence and control of various external cultures, at times involving the incursion of foreign populace. In 69 BC, Crete was annexed by the Romans and was passed on to the Byzantines almost five centuries later. The Arabs invaded the island in the year $824 \mathrm{AD}$. It was reconquered by the Byzantines in $961 \mathrm{AD}$. In spite of multiple local revolts, the latter period was followed by more than four centuries of Venetian rule that started in the early 1200 s. Then, after a two-decade siege by the Ottoman Empire of what is today the city of Heraklion, the Turks controlled the island from $1669 \mathrm{AD}$ until the early twentieth century, when it was unified with mainland Greece.

Although these changes in cultural orientation and human populations may have been concentrated initially in the coastal and lowland regions of Crete, the upland areas were not exempt from such external influences. For example, in eastern Crete, the population of the Lasithi Plateau in the Diktaian mountain range is also known to have been influenced to some extent by the same complex course of history. The first settlements of the Lasithi highland mesa have been dated to the later Neolithic, approximately $3500 \mathrm{BC}$, possibly as a result of population expansions from coastal homesteads. ${ }^{4}$ The archeological evidence suggests that, although they enjoyed a relative physical isolation, the inhabitants of the Lasithi Plain were, throughout its history, in communication with the inhabitants of the coastal regions.

The size of the Minoan population in the Lasithi Plateau appears to have reached a peak around 1600 BC and dropped sharply a couple of centuries later. The material culture of the latest Bronze Age displayed the hybrid Minoan/Mycenaean styles that were typical of this period in Crete. ${ }^{4}$ After another reduction in population density around the third century $\mathrm{BC}$, the area became populous again in the 4th century $\mathrm{AD}$, presumably as a result of a Roman settlement. ${ }^{4}$ During the period of Venetian rule, the Lasithi Plateau served as a refuge for Cretan insurgents. ${ }^{5}$ The Venetian government reacted by banning settlement and cultivation of the area from the mid 13th century to 1463 AD. ${ }^{4,5}$ Subsequently, in an attempt to increase grain production and alleviate the effects of the Ottoman blockade, the Venetian government decided to recolonize the plain in 1548 with settlers from the Greek mainland (eastern Peloponnese ${ }^{4,5}$ ). Although some have suggested that with every foreign intrusion into the Lasithi Plateau the local residents deserted the area, ${ }^{4}$ it remains entirely unclear as to what fraction, if any, of the local population departed in each period and the geographical origin(s) of those who remained.

A recent analysis of nine Y-chromosome markers defining haplogroups A, DE, G2, I, J, P*, and R1a revealed a high degree of heterogeneity within the island of Crete. ${ }^{6}$ Their study uncovered that, although more than $96 \%$ of males in the Cretan prefectures of Chania, Rethymno and Heraklion can be assigned to the tested haplogroups, only $82 \%$ of the Y chromosomes in Lasithi were derived at those markers. ${ }^{6}$ An analysis of $\mathrm{J} 2$ chromosomes from Crete, similarly revealed additional examples of diversity within the island. ${ }^{7}$

In the present study, we explore $\mathrm{Y}$ chromosomal diversity in the eastern half of Crete by typing a total of 77 markers in two general populations from the prefectures of Heraklion and Lasithi, and in a relatively isolated group from the Lasithi Plateau. The historical importance of the latter region is underscored by the role played by mountain plains, such as the one mentioned above, as natural refuges for late Minoans. ${ }^{3}$ The relative geographic isolation that has characterized the Lasithi Plateau even until the late twentieth century (the first and only paved road connecting the high plain with the outside world was built in the 1970s) provides a unique opportunity to study the genetic contributions of past migrants and invaders to the composition of the male population in this area, as compared with the more accessible locations of the island. Our results uncover significant frequency differences of the $\mathrm{J} 2$ and R1 haplogroups between the inhabitants of the isolated Lasithi Plateau and those of the surrounding Heraklion and Lasithi Prefectures. We demonstrate a nonuniform distribution of Y-chromosome signatures, which reflects the complex colonization of the eastern half of Crete by different mainland sources during the last 9000 years.

\section{Materials and methods Subjects}

A total of 168 unrelated male samples were collected from three locations in the island of Crete (Figure 1). Two sample groups of 104 and 23 individuals were from the general populations of the Heraklion and Lasithi Prefectures, respectively. A third group of 41 individuals was from the population of the Lasithi Plateau, a plain located within the Diktaian mountain range, at a median elevation of approximately 850 meters and encircled by $2000-m$-high mountains on all sides.

Individuals were identified, at the time of collection, by biographical information dating back to at least two generations. Samples were collected according to the guidelines of the Institutional Review Board at Florida 


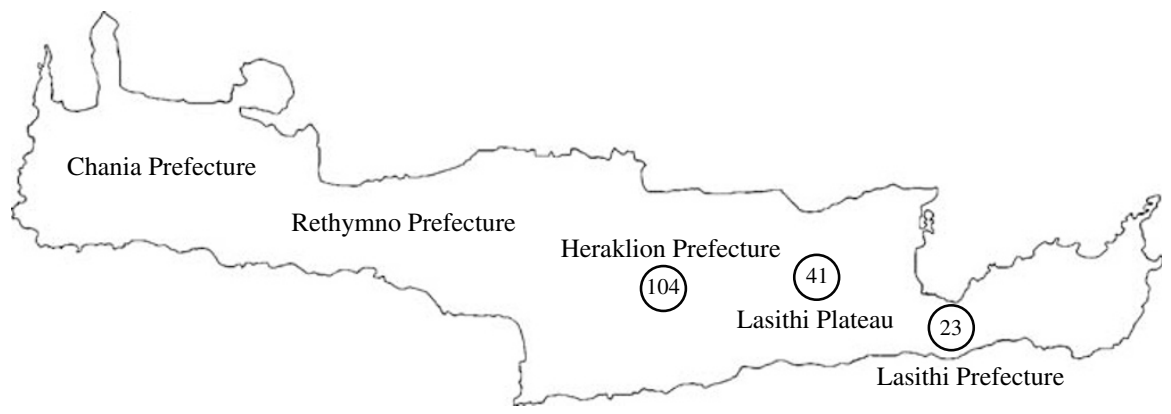

Figure 1 Geographic locations and number of samples of the three Cretan populations.

International University. Genomic DNA was isolated from peripheral blood lymphocytes by standard phenol/chloroform extraction followed by ethanol precipitation. ${ }^{8}$

\section{Y-chromosome haplotyping}

Seventy-seven binary markers were hierarchically genotyped by standard methods, including PCR-RFLP and size detection by PCR of the Y-specific polymorphic Alu insertion (PAI) YAP. For those polymorphisms not amenable to detection by RFLP, genotyping was performed by selective PCR. ${ }^{9,10}$ Supplementary Table 1 presents the summary of Y-SNPs genotyped for all the Cretan samples. Haplogroup diversity was calculated as $h=\left(1-\sum x_{i}^{2}\right) n /$ $(n-1) .{ }^{11}$ Information regarding the Y-chromosomal locations of the binary markers, the original references reporting their discoveries, the allelic states and the primer sequences can be found at the YCC website under Supplementary Data: http://ycc.biosci.arizona.edu/nomenclature_system/ frontpage.html.

\section{Correspondence analysis}

Y-chromosome data from 23 additional populations were obtained from the literature and were employed to perform a correspondence analysis (CA). Supplementary Table 2 presents the list of all the populations included in the study and their haplogroup frequencies. The CA was performed using the NTSYSpc-2.02i software ${ }^{12}$ and was based on the observed frequencies of haplogroups A, B, C, E3, ExE3, F* , G, H, I, J2, JxJ2, K-M70, KxM70, L, N, PxR, R*, and R1+R2 (Supplementary Table 2).

\section{Y-STR genotyping and age estimations of Y-STR variation}

For the Y-chromosome haplogroups R1a1-M198 and R1b-P25, Y-STR diversity at 10 markers (DYS19, DYS388, DYS389I, DYS389II, DYS390, DYS391, DYS392, DYS393, DYS439, and DYSA7.2) was assessed. The Y-STR data from the populations of the Heraklion and Lasithi Prefectures were combined and compared with the data from the Lasithi Plateau group. In addition, a total of 40 P25-derived samples from Ferrara, Italy, were also genotyped for the same 10 Y-STR markers.
Genotyping of Y-STR markers was performed as reported elsewhere $^{13}$ and the age estimations of Y-STR variation were calculated as previously described. ${ }^{14,15}$ For both the R1a1-M198 and the R1b-P25 haplogroups, the Cretan YSTR data were analyzed with respect to published Y-STR information from the Balkans ${ }^{16}$ and Turkey. ${ }^{13}$ Supplementary Table 3 presents the R1a1 and R1b-associated Y-STR data from all the populations that were analyzed. As Y-STR data on only seven markers were available for the Balkans (DYS19, DYS389I, DYS389II, DYS390, DYS391, DYS392, and DYS393), the age estimations of Y-STR variation were obtained separately by using both the 7- and 10-loci data sets (Table 1). BATWING expansion times were also generated for comparisons (Table 1), using an average STR mutation rate of 0.0007 , an intergeneration time of 25 years and an exponential population growth from a constant-size ancestral population. ${ }^{17}$ As mentioned above, the R1b-P25 analysis included data presented for the first time in this work from the population of Ferrara in northern Italy. Population-specific and overall network analyses were performed with 7 loci by using the reducedmedian algorithm of NETWORK 4.2.0.0 (Supplementary Figures 1 and 2). ${ }^{18}$ The Y-STR loci DYS19, DYS389I, DYS389II, DYS390, DYS391, DYS392, and DYS393 were assigned weights of $5,5,2,1,2,10$, and 10 , respectively. For each of these haplogroups, Y-STR-based principal component analyses (PCAs) were also generated using the 7-loci data sets (Figure 5). E3b1-M78 samples were genotyped for Y-STR markers A7.1, DYS19, and DYS439 in order to classify them into E3b1-M78 $\alpha, \beta, \gamma$, and $\delta$ lineages, respectively. ${ }^{19}$

\section{Results \\ Phylogeography}

From a total of 77 binary markers typed in the three Cretan populations, 35 were found to be polymorphic in the overall group of 168 individuals. These define 21 paternal haplogroups in the Heraklion Prefecture collection and 12 haplogroups in both the Lasithi Prefecture and the Lasithi Plateau groups (Figure 2). Only seven of these 12 haplogroups are observed in common in the latter two 
Table 1 Ages of Y-STR variation and expansion times for lineages R1a1 and R1b

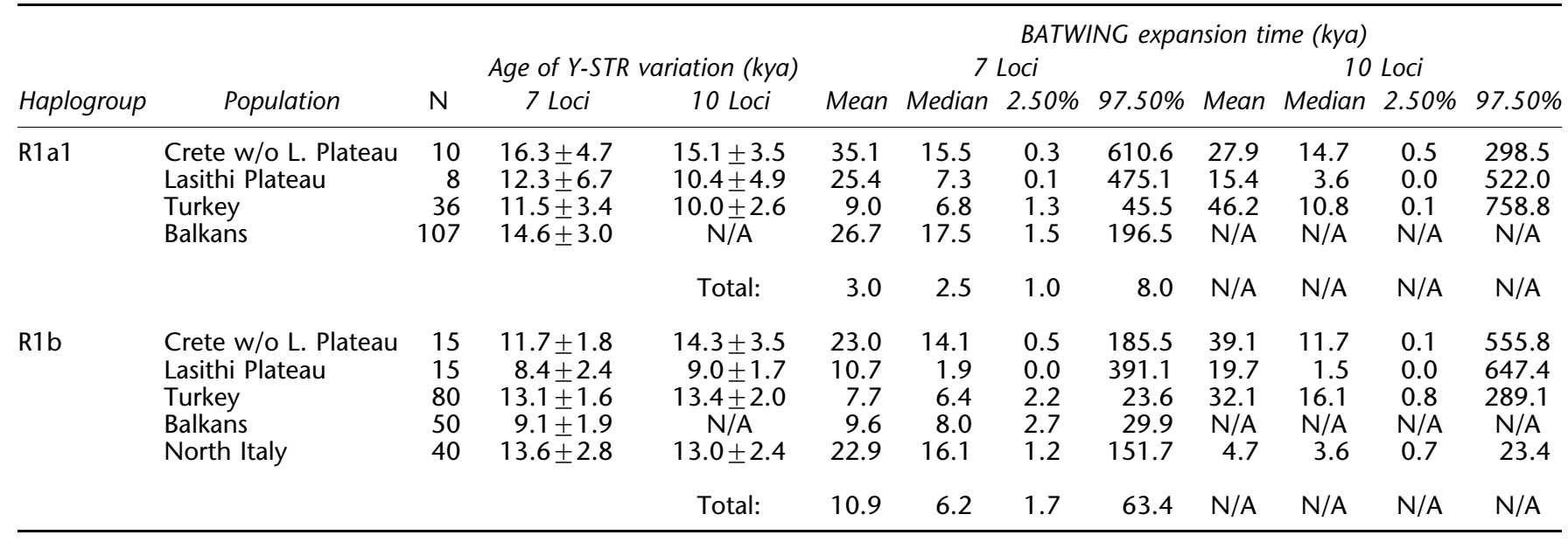
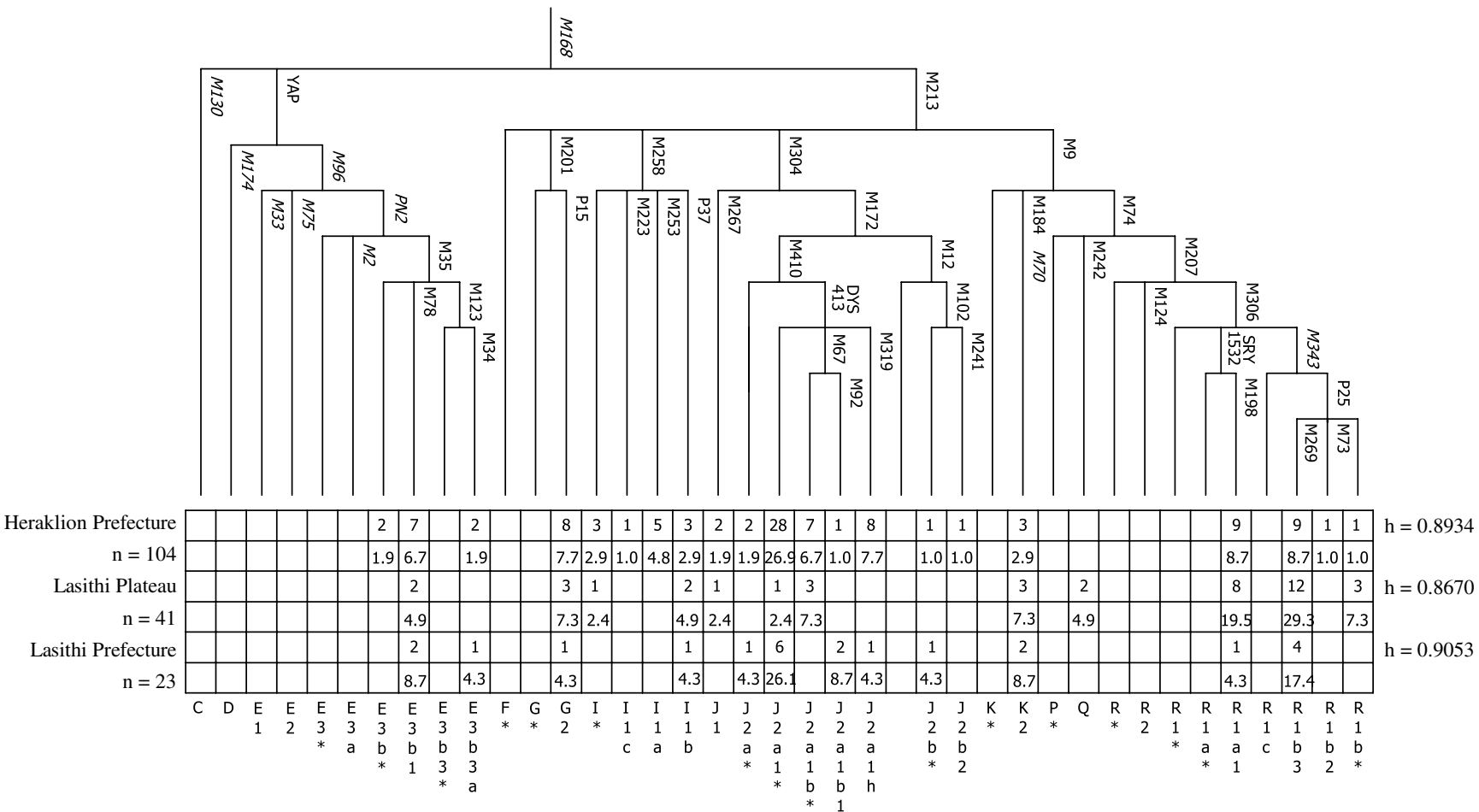

Figure 2 Most parsimonious phylogeny of Y-chromosome haplogroups. The number of samples derived at each haplogroup, frequencies and haplotype diversity $(\mathrm{h})$ are indicated for each population. All M78-derived individuals belonged to the E3b1-M78 $\alpha$ classification, except for two samples in the Heraklion Prefecture population and one from the Lasithi Plateau, who were M78 $\delta$. Mutations in italics were not genotyped and are only included for phylogenetic context. Markers M3, M18, M20, M21, M25, M26, M37, M56, M65, M68, M72, M81, M120, M123, M136, M137, M148, M153, M157, M158, M161, M163, M167, M204, M222, M224, M227, M231, M280, M281, M285, M286, M289, M290, M318, M320, M321, M322, M323, M339, M340, M365, M368, M369, and V6 were found to have the ancestral state in all individuals genotyped.

populations from the Lasithi region. With the exception of two Q-M242 individuals from the Lasithi Plateau, the rest of the haplogroups detected in the Lasithi Prefecture and Lasithi Plateau collections were also observed in the province of Heraklion.
In terms of haplogroup frequencies, the most striking differences among the three populations studied are observed in the J2 and R1 haplogroups. Although 46.2 and $47.8 \%$ of the individuals in the Heraklion Prefecture and Lasithi Prefecture populations, respectively, are derived 
at M172, a frequency of only $9.8 \%$ is found in the Lasithi Plateau group. Furthermore, not only does the highland plain record a much lower percentage of $\mathrm{J} 2$ individuals, but it is also characterized by a lower level of diversity. While a total of seven and five $\mathrm{J} 2$ haplogroup types are detected in the Heraklion Prefecture and Lasithi Prefecture populations, respectively, only two are observed in the Lasithi Plateau group. In the case of the R1 haplogroup, while frequencies of $19.2 \%$ and $21.7 \%$ are found in the Heraklion Prefecture and Lasithi Prefecture populations, respectively, more than half $(56.1 \%)$ of the Lasithi Plateau individuals are R1-M306-derived.

When such haplogroup distributions are compared with those previously observed in other Mediterranean locations, $^{20-24}$ it becomes evident that the low $\mathrm{J} 2$ frequency in the Lasithi Plateau population is exceptional in the northeastern region of the Mediterranean (Figure 3). In this area, only two collections, from Macedonia and Syria, have J2 frequencies that come close to the one observed in the Lasithi Plateau (Figure 3). Equally discordant is the high frequency of the R1 haplogroup in the Lasithi Plateau group when compared to other groups in the northeastern Mediterranean. Overall, the proportion of R1 individuals found in the Lasithi Plain is actually more equivalent to the frequencies detected in northwestern Mediterranean locations, such as central-northern Italy, Corsica, mainland France and Spain (Figure 3).

In contrast with the results of the above comparisons, both the Heraklion Prefecture and Lasithi Prefecture populations present J2 and R1 frequencies comparable with those previously observed in northeastern Mediterranean locations and even in Libya (Figure 3). Nevertheless, the frequency of J2 in these two Cretan groups is much higher than in mainland Greece, Macedonia and Albania.

\section{Population relationships}

In addition to the three Cretan populations examined in this work, Y-chromosome data from 23 Mediterranean groups were obtained from the literature (Supplementary Table 2) and were used to perform a CA (Figure 4). These same populations are included in Figure 3 for a visual representation of Y-haplogroup distributions in the Mediterranean region.

The CA illustrates very close genetic relationships between three pairs of populations (Figure 4). These are the Heraklion Prefecture and Lasithi Prefecture groups, the populations from the islands of Corsica and Sardinia, and the groups from Albania and mainland Greece. All of the above populations, in turn, appear closely related to those of Sicily, Calabria and Turkey. Furthermore, although representing a slightly higher degree of separation, the collections from Syria and Lebanon segregate close to the latter group of populations.

In contrast with the positions of the other two Cretan collections, the Lasithi Plateau population exhibits a close genetic relationship with respect to groups from central and southwestern Europe, including Andalusia, Basque Country, Cataluña, Croatia, France and central-northern Italy (Figure 4).

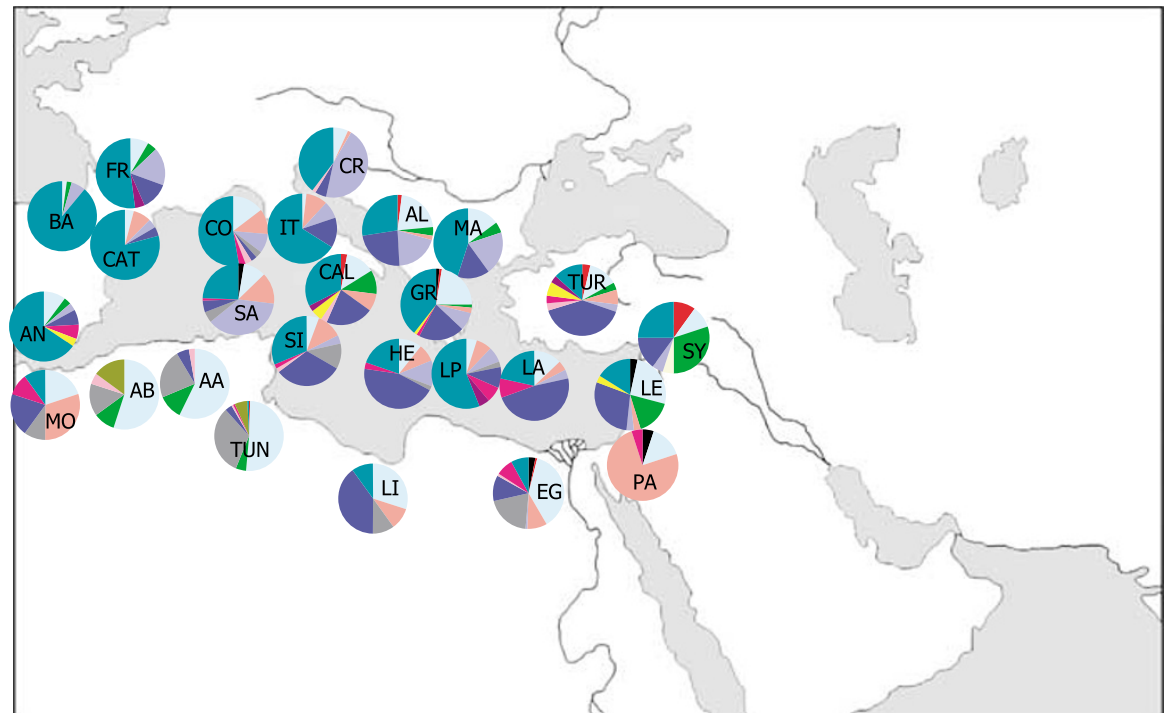

\begin{tabular}{ll|l|l|l|l|l|l|l|l|l|l|l|l|l}
\hline $\mathrm{A}+\mathrm{B}+\mathrm{C}$ & ExE3 & E3 & $\mathrm{F}$ & $\mathrm{G}$ & $\mathrm{H}$ & $\mathrm{I}$ & $\mathrm{J} \times \mathrm{J} 2$ & $\mathrm{~J} 2$ & $\mathrm{~K} \times \mathrm{M} 70$ & $\mathrm{~K}-\mathrm{M} 70$ & $\mathrm{~L}+\mathrm{N}$ & $\mathrm{P} \times \mathrm{R}$ & $\mathrm{R}^{*}$ & $\mathrm{R} 1+\mathrm{R} 2$
\end{tabular}

Figure 3 Distribution of Y-chromosome haplogroups in 26 Mediterranean populations. Original references, number of samples and name codes for all populations are listed in Supplementary Table 2. 


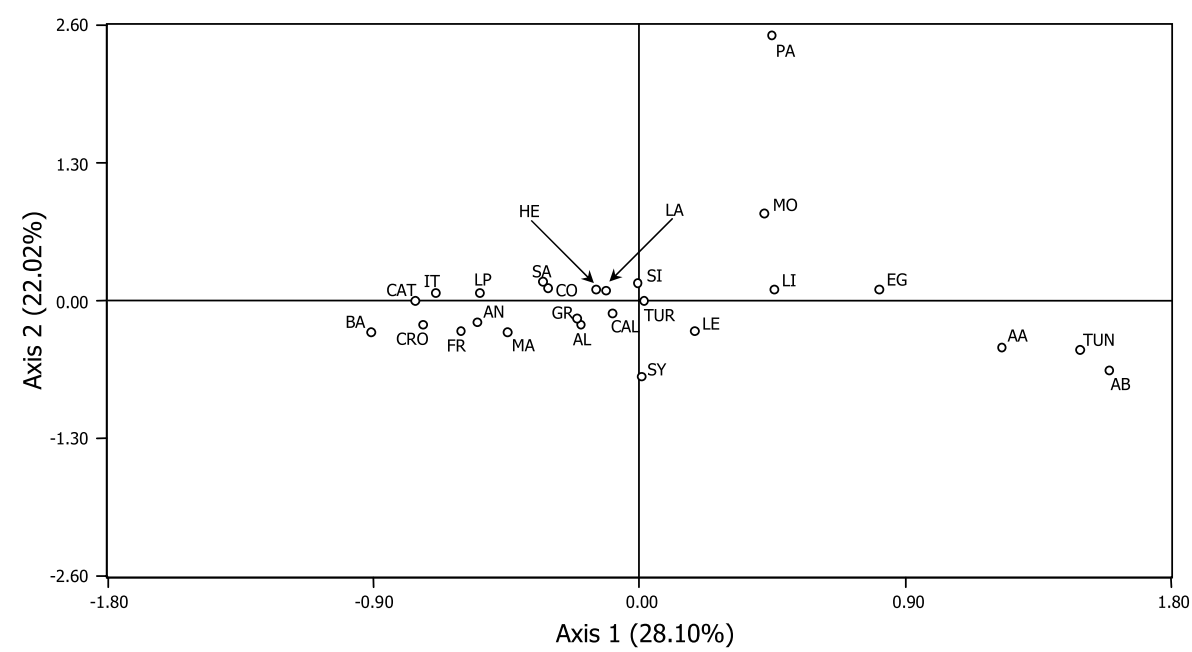

Figure 4 Correspondence analysis of 26 Mediterranean populations based on the observed frequencies of haplogroups $A, B, C, E 3, E x E 3, F *, G, H$, $\mathrm{I}, \mathrm{J} 2, \mathrm{~J} \times \mathrm{J} 2, \mathrm{~K}-\mathrm{M} 70, \mathrm{~K} \times \mathrm{M} 70, \mathrm{~L}, \mathrm{~N}, \mathrm{P} \times \mathrm{R}, \mathrm{R}^{*}$, and $\mathrm{R} 1+\mathrm{R} 2$. Original references, number of samples and name codes for all populations are listed in Supplementary Table 2.

\section{Y-STR genotyping and age estimations}

Microsatellite haplotypes based on 10 Y-STR markers were assessed for individuals belonging to the haplogroups R1a1-M198 and R1b-P25 (Supplementary Table 3). The ages of Y-STR variation for the Heraklion Prefecture and Lasithi Prefecture collections were estimated by combining Y-STR data from both groups. The results are compared to the ages determined for the Lasithi Plateau population, the Balkans, ${ }^{16}$ Turkey $^{13}$ and North Italy (i.e., Ferrara) (Table 1).

Table 1 indicates the age of Y-STR variation associated with each haplogroup for every one of the populations examined. BATWING expansion times are also included for comparison. As Y-STR information for only seven markers was available in the case of the Balkans, microsatellitebased dating was performed separately using both the 7-loci and 10-loci data sets. The results indicate that for the R1a1 haplogroup, the age of Y-STR variation in the Lasithi Plateau is at least $10.4 \pm 4.9 \mathrm{kya}$, the same range as in the case of Turkey $(10.0 \pm 2.6$, Table 1). Nevertheless, the ages of Y-STR variation in both of these populations are more recent than the dates estimated for the Balkans $(14.6 \pm 3.0 \mathrm{kya})$ and the rest of Crete (at least $15.1 \pm 3.5 \mathrm{kya})$. In the case of the R1b haplogroup, the age of Y-STR diversity in the Lasithi Plateau population is at least $8.4 \pm 2.4 \mathrm{kya}$, compared to at least $11.7 \pm 1.8 \mathrm{kya}$ in the surrounding two provinces. The former date is comparable with the age estimated for the Balkans (Table 1). Unfortunately, for many of the populations analyzed and for all groups from Crete, the BATWING expansion analyses did not generate credible 95\% confidence intervals (Table 1). In spite of that, with the exception of the R1b results for the Lasithi Plateau and Turkey, the times of expansion corroborate the above-mentioned ages.

The population-specific network analyses generated star-like topologies in most of the groups analyzed
(Supplementary Figure 1). In addition, the overall networks for haplogroups R1a1 and R1b demonstrate that all the collections have representative haplotypes in, presumably, the most ancestral node of their topologies (Supplementary Figure 2). This explains the relatively old dates and the similar ages of Y-STR variation estimated for all groups studied.

In order to explore the genetic similarities of the R1a1 and R1b Cretan haplogroups with those from Turkey, the Balkans and North Italy, Y-STR-based PCAs based on seven microsatellite markers, were generated. These PCAs are presented in Figure 5. The plot in panel A indicates a close genetic relationship between the Lasithi Plateau and the Balkans R1a1 lineages, whereas the Crete without the Lasithi Plateau group exhibits little or no affinity with respect to R1a1 lineages from the former populations and from Turkey (Figure 5a). In the case of the R1b haplogroup (Figure $5 \mathrm{~b}$ ), the PCA indicates that R1b lineages from both Cretan groups are more related to the lineages from North Italy than to those from Turkey or the Balkans. This affinity, however, is much more evident in the case of the Crete-without the Lasithi Plateau population.

\section{Discussion}

Neolithic and postneolithic signals from eastern Crete The clinal frequency pattern of haplogroup $\mathrm{E}$ in the Mediterranean region has been associated with several dispersal events during and after the Neolithic period. ${ }^{19,25,26}$ Originally emanating from East Africa, haplogroup E3b-M35 and particularly subhaplogroup E3b1M78 are believed to be signatures of a demic diffusion of Neolithic farmers from the Middle East to Europe. This haplogroup has left its strongest imprint along the Southern Mediterranean. ${ }^{20,26,27}$ 
a $\quad$ R1a1
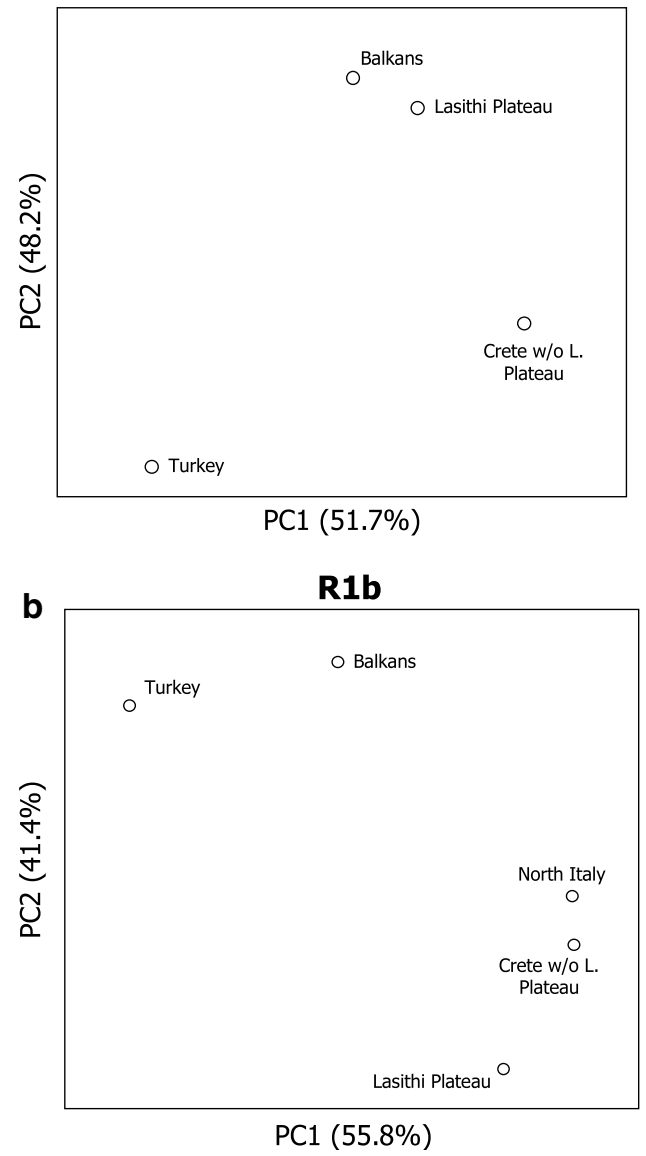

Figure 5 Y-STR-based principal component analyses of haplogroups R1a1 and R1b using microsatellite markers DYS19, DYS389l, DYS389II, DYS390, DYS391, DYS392, and DYS393.

In the three Cretan populations studied in this work, the frequency of E3b-M35-derived chromosomes range from $4.9 \%$ in the Lasithi Plateau to $13 \%$ in the Lasithi Prefecture population. As expected, the most common subhaplogroup observed is E3b1-M78, which is the only one found in the Lasithi Plateau collection (Figure 2). Further genotyping of the M78-derived samples for the Y-STR markers A7.1, DYS19 and DYS439, allowed their classification into two of the four previously delineated E3b1-M78 lineages (ie, $\alpha, \beta, \gamma$, and $\delta$ ). ${ }^{19}$ One chromosome from the Lasithi Plateau population and two chromosomes from the Heraklion Prefecture belong to the lineage $M 78 \delta$, whereas the remaining eight Cretan samples belong to the lineage $M 78 \alpha$. The highest frequency of the M78 $\alpha$ lineage was previously observed in the Balkans. This decreased in the western and northeastern directions in European populations. ${ }^{19}$ Cluster $\mathrm{M} 78 \delta$, on the other hand, was detected at low frequencies in Europe, the Near East, eastern and northern Africa, ${ }^{19}$ and it has been found to be associated with at least three M78 downstream mutations. ${ }^{28}$ Given the paraphyletic character of this cluster, it is not possible to relate, at this juncture, the Cretan M78 $\delta$ chromosomes with those found in neighboring locations. Considering the relatively high frequency of E3b1-M78 chromosomes in Albania, Macedonia and Greece, ${ }^{16,19,26}$ and particularly the $M 78 \alpha$ lineage in the first two of these collections (data not available for Greece), it is possible that the presence of this lineage in Crete represents gene flow from mainland Greece during and/or after the Neolithic.

In the case of Cretan E3b3-M123 (M34) chromosomes, they most likely signal East African or Middle-Eastern gene flow rather than European, due to the scarcity of this lineage in the latter area. ${ }^{19,26}$ Similarly, the presence of E3b-M35* individuals in the Heraklion Prefecture population could probably be attributed to an East-African or North-African contribution.

Like haplogroup $\mathrm{E}$, the entire $\mathrm{J}$ clade is also of Middle Eastern origin. It was most likely introduced into the Mediterranean region during the diaspora of Neolithic farmers. ${ }^{20,25,26}$ It has been suggested that this dispersal may have occurred overseas, ${ }^{25,26}$ which implies a significant role of the Mediterranean islands in the Neolithization of southern continental Europe.

An analysis of J2-frequency clines in both continental Italy and in Greece, as well as in the islands of Crete, Mitilini and Khios, revealed high frequency values of J2chromosomes in all the four Cretan prefectures (26-45\%) with the lowest corresponding to Lasithi. ${ }^{6}$ Our study corroborates the predominant frequency of the J2 haplogroup in the Heraklion and Lasithi Prefecture populations (46.2 and $47.8 \%$, respectively), which are most comparable with the values reported for Turkey ${ }^{20}$ and Libya ${ }^{24}$ (Figure 3). In the case of the latter, however, the majority of J2 chromosomes correspond to the J2b2-M241 subhaplogroup. This correspondence suggests little genetic affinity with the Cretan collections.

Out of the total number of J2 chromosomes in the Heraklion Prefecture and Lasithi Prefecture populations, 92 and $82 \%$ of the samples, respectively, are derived at J2DYS413 (Figure 2). As the CA illustrates (Figure 4), these proportions suggest genetic affinity with groups from the Turkish-Greek area, where this marker is believed to have originated. ${ }^{7}$ Based on the generally low frequency of J2DYS413 chromosomes in present mainland Greece, ${ }^{6}$ the Turkish region is favored as the origin of these lineages. Furthermore, the high frequencies of DYS413-derived chromosomes in the island of Crete, as well as in a number of coastal locations from both mainland Greece and Italy, together with the moderate frequencies of this mutation in the Aegean islands, ${ }^{6}$ can be interpreted as the signals of a maritime demic diffusion from Anatolia.

A previous study of DYS413-derived chromosomes from the Mediterranean region also detected significant similarities between Cretan and southern Anatolian populations. These findings corroborated the proposed geographical 
origin of the earliest Cretan agriculturists. ${ }^{29}$ Nevertheless, considering the number of colonizations experienced by the island in more recent periods (including the Ottoman penetration), the presence of DYS413 chromosomes in Crete may not be necessarily solely related to the arrival of its first farmers.

Of the 57 J2-DYS413 chromosomes in Crete, a total of nine chromosomes are derived at J2a1h-M319 (Figure 2). This mutation was recently discovered in one Iraqi and two Moroccan chromosomes ${ }^{24}$ and to our knowledge, it has not been reported in any other population. Interestingly, seven out of the nine Cretan M319-derived chromosomes have the (CA)16-(CA)18 genotype at the DYS413 microsatellite marker, a pattern that was not observed in any other DYS413-derived lineage. A prominent frequency of DYS413 (CA)16-(CA)18 chromosomes in Crete, and particularly in the prefecture of Chania, was detected in the past and was attributed to a founder effect in the island. ${ }^{7}$ It appears from our results that this founder effect is associated with the introduction or origin in Crete of the J2a1h-M319 mutation.

Archeological findings indicate that the first inhabitants of Crete arrived during the Neolithic. ${ }^{1}$ It is therefore intriguing that the Lasithi Plateau population only presents $12.2 \%$ of $\mathrm{J}$ chromosomes, compared with values of more than $47 \%$ in both the Heraklion Prefecture and Lasithi Prefecture collections. Similarly, only $4.9 \%$ of chromosomes in the Lasithi Plateau group correspond to the E haplogroup, compared to more than $10 \%$ in the former two populations. The low frequencies of these Neolithic haplogroups in the Lasithi Plateau population contrast with the unusually elevated proportion of Paleolithic R1 chromosomes for an eastern Mediterranean location.

\section{Haplogroup R1 chromosomes in eastern Crete}

Previous studies of Y-chromosome polymorphisms in Eurasian populations have suggested a Paleolithic origin for the Y-haplogroup R1. ${ }^{20,30}$ The origin of the haplogroup R1-M173 is believed to predate the Last Glacial Maximum. This haplogroup dispersed from east to west, possibly $30 \mathrm{kya}$, along with the spread of the Aurignacian culture. ${ }^{20,30}$ Different demographic processes have been proposed to account for the current distributions of subclades R1a and R1b in European populations. ${ }^{16,20,30,31}$

The R1 haplogroup frequencies obtained for the three Cretan groups in the present work reveal remarkable differences among them, as well as with respect to neighboring Mediterranean collections. Although frequencies of 19.2 and $21.7 \%$ were found in the Heraklion Prefecture and Lasithi Prefecture populations, respectively, a proportion of more than half $(56.1 \%)$ was observed in the Lasithi Plateau. The prominent frequency of the R1 haplogroup in the Lasithi Plateau collection does not resemble the values published for any other group in the northeastern Mediterranean. Although this high proportion of R1 chromosomes in the Lasithi high plain could be the result of genetic drift owing to founder effect and/or to geographic isolation, the above observation prompted us to explore the possible origins and ages of the R1 haplogroups in the island.

Similar ages of R1a1-associated Y-STR variation were obtained for all the populations analyzed (Table 1). These results are indicative of considerable microsatellite variability associated with this haplogroup in the island. The Y-STR-based PCA of Figure 5a indicates a very close genetic relationship between the R1a1 chromosomes from the Lasithi Plateau and those from the Balkans. In contrast, the rest of the R1a1 chromosomes from Crete (denoted as 'Crete without Lasithi Plateau' in Figure 5a) do not appear closely related to those from the Lasithi Plateau, Turkey or the Balkans. These results suggest that the R1a1 chromosomes in the highland mesa may represent a unique infiltration into Crete, distinct from the incursion of R1a1 types into the coastal regions.

In the case of the R1b haplogroup, the age of Y-STR variation estimated for the Crete without Lasithi Plateau population is similar to the dates obtained for Turkey and North Italy (Table 1). The Y-STR-based PCA of this haplogroup (Figure 5b) indicates a close genetic relationship between the Crete without Lasithi Plateau collection and North Italy. The Lasithi Plateau group segregates at a slightly more distant point in the PCA plot, with respect to North Italy. Based on the age of the R1b-associated Y-STR variation for the Crete-without-Lasithi-Plateau population, the genetic affinity between R1b haplogroups from North Italy and Crete might be the imprints of an Italian gene flow before the end of the Minoan civilization and/or more recent migrations during the Roman and Venetian ruling periods. Finally, it is possible that the more recent age for the R1b-associated Y-STR variation in the Lasithi Plateau population as compared with the estimate for the rest of eastern Crete could have resulted from population bottlenecks in the mountain plain. Alternatively, these lineages might have been introduced to the Lasithi highland plain long after their presence in other regions of the island.

\section{Conclusions}

The genetic complexity of the male population in Crete is as fascinating as the history of the island. The diversity of Y-chromosome haplogroups ascertained in the three Cretan groups that were analyzed, and particularly in the Heraklion Prefecture population, reflects the genetic legacy of multiple migrations over the last 9000 years. Equally significant is the predominant frequency of Paleolithic haplogroups in the Lasithi Plateau, which includes rare chromosomes such as I* and Q-M242 and is perhaps an indication of the prehistorical complexity of 
this population. The differences in Y-haplogroup frequencies between the Lasithi Plateau collection and the other two Cretan groups analyzed are either due to genetic drift favored by geographic isolation and/or to the replacement by more recent migrations of a Lasithi Plateau-like genetic composition in the other two Cretan populations. As a whole, our results may be indicative of the utilization of the Lasithi highland retreat as a long-standing repetitive pre-Minoan refugium.

\section{Acknowledgements}

We gratefully acknowledge Dr Manuela M Regueiro for her technical assistance.

\section{References}

1 Broodbank C, Strasser T: Migrant farmers and the Neolithic colonisation of Crete. Antiquity 1991; 65: 233-245.

2 Fitton JL: Minoans. London: The British Museum Press, 2002.

3 Nowicki K: Defensible sites in Crete c1200 - 800 BC (LMIIIB/IIIC through Early Geometric). Université de Liège, Liège, University of Texas, Austin: Aegaeum Series 21, 2000.

4 Watrous LV: Lasithi: a history of settlement on a highland plain in Crete. Hesperia Supplement 1982; 18: i-iii+v+vii-xiv+1-89+91101+103-122.

5 Rackham $\mathrm{O}$, Moody J: The making of the Cretan landscape. Manchester: Manchester University Press, 1996.

6 Di Giacomo F, Luca F, Anagnou N et al: Clinal patterns of human $\mathrm{Y}$ chromosomal diversity in continental Italy and Greece are dominated by drift and founder effects. Mol Phylogenet Evol 2003; 28: 387-395.

7 Malaspina P, Tsopanomichalou M, Duman T et al: A multistep process for the dispersal of a $\mathrm{Y}$ chromosomal lineage in the Mediterranean area. Ann Hum Genet 2001; 65: 339-349.

8 Sambrook J, Russell DW: Molecular cloning: a laboratory manual. New York: Cold Spring Harbor Laboratory Press, 2001.

9 Martinez L, Reategui EP, Fonseca LR et al: Superimposing polymorphism: the case of a point mutation within a polymorphic Alu insertion. Hum Hered 2005; 59: 109-117.

10 Regueiro M, Cadenas AM, Gayden T, Underhill PA, Herrera RJ: Iran: tricontinental nexus for Y-chromosome driven migration. Hum Hered 2006; 61: 132-143.

11 Nei M, Tajima F: DNA polymorphism detectable by restriction endonucleases. Genet 1981; 97: 145-163.

12 Rohlf F: NTSYSpc. Setauket, New York: Exeter Publishing, 2002.

13 Cinnioglu C, King R, Kivisild T et al: Excavating Y-chromosome haplotype strata in Anatolia. Hum Genet 2004; 114: 127-148.

14 Zhivotovsky LA: Estimating divergence time with the use of microsatellite genetic distances: impacts of population growth and gene flow. Mol Biol Evol 2001; 18: 700-709.

15 Zhivotovsky LA, Underhill PA, Cinnioglu C et al: The effective mutation rate at $\mathrm{Y}$ chromosome short tandem repeats, with application to human population-divergence time. Am J Hum Genet 2004; 74: 50-61.

16 Perièiæ M, Lauc LB, Klaric IM et al: High-resolution phylogenetic analysis of southeastern Europe traces major episodes of paternal gene flow among Slavic populations. Mol Biol Evol 2005; 22 : $1964-1975$.

17 Wilson I, Weale M, Balding D: BATWING: Bayesian analysis of trees with internal node generation. Aberdeen: Department of Mathematical Sciences, University of Aberdeen, 2000.

18 Rohl A: Network 2.0: a program package for calculating phylogenetic networks. Mathematisches Seminar, Hamburg, Germany: University of Hamburg, 1997.

19 Cruciani F, La Fratta R, Santolamazza P et al: Phylogeographic analysis of haplogroup E3b (E-M215) Y chromosomes reveals multiple migratory events within and out of Africa. Am J Hum Genet 2004; 74: 1014-1022.

20 Semino O, Passarino G, Oefner PJ et al: The genetic legacy of Paleolithic Homo sapiens sapiens in extant Europeans: a Y chromosome perspective. Science 2000; 290: 1155-1159.

21 Francalacci P, Morelli L, Underhill PA et al: Peopling of three Mediterranean islands (Corsica, Sardinia, and Sicily) inferred by Y-chromosome biallelic variability. Am J Phys Anthropol 2003; 121: 270-279.

22 Arredi B, Poloni ES, Paracchini S et al: A predominantly Neolithic origin for Y-chromosomal DNA variation in North Africa. Am J Hum Genet 2004; 75: 338-345.

23 Luis JR, Rowold DJ, Regueiro M et al: The Levant versus the Horn of Africa: evidence for bidirectional corridors of human migrations. Am J Hum Genet 2004; 74: 532-544.

24 Shen P, Lavi T, Kivisild T et al: Reconstruction of patrilineages and matrilineages of Samaritans and other Israeli populations from Ychromosome and mitochondrial DNA sequence variation. Hum Mutat 2004; 24: 248-260.

25 Di Giacomo F, Luca F, Popa LO et al: Y chromosomal haplogroup J as a signature of the post-neolithic colonization of Europe. Hum Genet 2004; 115: 357-371.

26 Semino O, Magri C, Benuzzi G et al: Origin, diffusion, and differentiation of Y-chromosome haplogroups $\mathrm{E}$ and J: inferences on the neolithization of Europe and later migratory events in the Mediterranean area. Am J Hum Genet 2004; 74: 1023-1034.

27 Hammer MF, Karafet T, Rasanayagam A et al: Out of Africa and back again: nested cladistic analysis of human Y chromosome variation. Mol Biol Evol 1998; 15: 427-441.

28 Cruciani F, La Fratta R, Torroni A, Underhill PA, Scozzari R: Molecular dissection of the Y chromosome haplogroup E-M78 (E3b1a): A posteriori evaluation of a microsatellite-network-based approach through six new biallelic markers. Hum Mutat 2006; 27: $831-832$.

29 Malaspina P, Cruciani F, Santolamazza P et al: Patterns of malespecific inter-population divergence in Europe, West Asia and North Africa. Ann Hum Genet 2000; 64: 395-412.

30 Wells RS, Yuldasheva N, Ruzibakiev R et al: The Eurasian heartland: a continental perspective on Y-chromosome diversity. Proc Natl Acad Sci USA 2001; 98: 10244-10249.

31 Marjanovic D, Fornarino S, Montagna S et al: The peopling of modern Bosnia-Herzegovina: Y-chromosome haplogroups in the three main ethnic groups. Ann Hum Genet 2005; 69: 757-763.

Supplementary Information accompanies the paper on European Journal of Human Genetics website (http://www.nature.com/ejhg) 